\title{
Evolutionary dynamics of a conserved sequence motif in the ribosomal genes of the ciliate Paramecium
}

\author{
Francesco Catania*, Michael Lynch
}

\begin{abstract}
Background: In protozoa, the identification of preserved motifs by comparative genomics is often impeded by difficulties to generate reliable alignments for non-coding sequences. Moreover, the evolutionary dynamics of regulatory elements in $3^{\prime}$ untranslated regions (both in protozoa and metazoa) remains a virtually unexplored issue.

Results: By screening Paramecium tetraurelia's 3' untranslated regions for 8-mers that were previously found to be preserved in mammalian 3' UTRs, we detect and characterize a motif that is distinctly conserved in the ribosomal genes of this ciliate. The motif appears to be conserved across Paramecium aurelia species but is absent from the ribosomal genes of four additional non-Paramecium species surveyed, including another ciliate, Tetrahymena thermophila. Motif-free ribosomal genes retain fewer paralogs in the genome and appear to be lost more rapidly relative to motif-containing genes. Features associated with the discovered preserved motif are consistent with this 8-mer playing a role in post-transcriptional regulation.

Conclusions: Our observations 1) shed light on the evolution of a putative regulatory motif across large phylogenetic distances; 2) are expected to facilitate the understanding of the modulation of ribosomal genes expression in Paramecium; and 3) reveal a largely unexplored-and presumably not restricted to Parameciumassociation between the presence/absence of a DNA motif and the evolutionary fate of its host genes.
\end{abstract}

\section{Background}

Conserved motifs in 3' untranslated regions (UTRs) have been identified in several organisms, including vertebrates [1,2], Drosophila [3-6], nematodes [6,7], S. cerevisiae [8], plants [9] and protozoa [10,11]. The function of these preserved sequences has often been revealed and may vary, being for example associated with cytoplasmic localization of the motif-containing mRNA and/or modulation of gene expression level. The regulatory aspect of conserved 3' UTR sequences has received large attention in recent years, and it has become apparent that 3' UTRs motifs often play a major role in post-transcriptional regulation [12], via their binding with microRNAs (miRNAs) [2,13]. Despite these crucial functions and some significant efforts to catalogue 3' UTR signals $[2,8]$, we still know relatively little of the evolutionary dynamics of these cis-regulatory sequences.

\footnotetext{
* Correspondence: fcatania@indiana.edu

Department of Biology, Indiana University, 1001E $3^{\text {rd }}$ Street, Bloomington, IN 47405, USA
}

In this article, we report the discovery of a conserved motif in the 3' UTRs of genes in the ciliate Paramecium tetraurelia and describe a number of features associated with this sequence (e.g., presence/absence across nonParamecium species, evolutionary fate of the genes that carry the motif in their 3' UTR, and putative association with miRNAs). The detected motif-GUACAUUA-and a number of its variants are also conserved in mammalian 3' UTRs [2]. However, while no particular association with any gene class is reported for mammals, in P. tetraurelia both GUACAUUA and several of its degenerate variants are most frequently contained in the 3' UTRs of ribosomal protein genes.

\section{Results}

Previously described mammalian 3' UTR motifs are found in the $3^{\prime}$ UTRs of $P$. tetraurelia ribosomal protein genes

We surveyed $P$. tetraurelia 3' UTRs for the presence of conserved motifs. To this end, we did not take advantage of a motif-discovery computer program, instead we 
Table 1 The number of hit 3' UTRs and the most frequent common function of the host genes are shown for the each of the most recurrent sequence motifs.

\begin{tabular}{llccc}
\hline & $\begin{array}{c}\text { Sequence } \\
\text { motif }\end{array}$ & $\begin{array}{c}\text { Observed } \\
\text { hits }\end{array}$ & $\begin{array}{c}\text { Expected } \\
\text { hits }\end{array}$ & Protein function* \\
\hline $\mathbf{1}$ & GUACAUUA & 127 & $13(3.67)$ & Ribosomal (88.5\%) \\
$\mathbf{2}$ & UGUACAUU & 47 & $11(3.12)$ & Ribosomal (61.5\%) \\
$\mathbf{3}$ & ACAAUCAU & 36 & $15(3.52)$ & - \\
$\mathbf{4}$ & UAUGCAAA & 35 & $12(3.82)$ & - \\
$\mathbf{5}$ & UUAUGCAA & 34 & $12(4.25)$ & - \\
$\mathbf{6}$ & AUGUACAU & 33 & $12(3.30)$ & Ribosomal (72.4\%) \\
$\mathbf{7}$ & UUUAUGCA & 32 & $12(3.65)$ & - \\
$\mathbf{8}$ & AUAUGCAA & 30 & $14(4.21)$ & - \\
$\mathbf{9}$ & UGUACAAU & 30 & $12(3.16)$ & Ribosomal (65.2\%) \\
$\mathbf{1 0}$ & GUACAUUU & 29 & $12(3.90)$ & Ribosomal (77.8\%) \\
$\mathbf{1 1}$ & UUGCAAUA & 29 & $13(3.37)$ & - \\
$\mathbf{1 2}$ & UAUGCAAU & 25 & $13(3.25)$ & - \\
$\mathbf{1 3}$ & UAUGUACA & 25 & $13(3.49)$ & Ribosomal (61.1\%) \\
\hline
\end{tabular}

Sequences that overlap the most abundant motif (GUACAUUA) are underlined. The expected average number of hits (standard deviation is in parentheses) is obtained by screening 25 sets of randomly generated DNA sequences, whose length and nucleotide composition are identical to the 3 UTRs sequences of the original dataset. ${ }^{*}=$ estimate based on genes with annotated function

initially screened the untranslated regions for a set of 8 mers that have been found to be conserved in mammalian 3' UTRs [2]. We found that 384 (71.1\%) of these motifs hit the set of the Paramecium 3' UTR sequences, with the vast majority of the 8-mers yielding only one or a few hits $(81 \%$ yielded $\leq 10$ hits). Among the motifs that most frequently hit the Paramecium 3' UTRs (Table 1), several share sequence similarity and partially overlap with the motif GUACAUUA, which yielded the highest number of hits, being detected in 127 3' UTRs.

The analysis of the function of the genes containing the most recurrent motifs revealed a pronounced association with ribosomal genes (Table 1). 100 out of 113 genes with annotated functions that contain the sequence GUACAUUA code for ribosomal protein-coding genes (the motif is additionally found in the 3' UTRs of two likely ribosomal genes that are annotated as part of putative chimeric transcription units in the $P$. tetraurelia genome, GSPATP00006003001 and GSPATP00004104001). In these genes, the start of the GUACAUUA motif is positioned at an average distance of $18.95 \mathrm{bp}(\mathrm{SD}=5.12)$ from the translation termination codon. The function of the 11 remaining genes is provided in Table 2; most of these genes code for proteins that are membrane-associated or involved in membrane biogenesis, and for factors that participate in transcription, RNA processing and regulation, and translation.

We collected all genes that code for ribosome-associated proteins in P. tetraurelia $(\mathrm{n}=472$, on the basis of BLAST best hits against the UNIPROT database), and used the program MEME to screen their 3' UTRs, to verify both that the most likely width of the conserved motif in the ciliate is eight bases as in mammals and that these 3' UTRs contain no common motifs other than those we detected. The motif-discovering software found a single, highly conserved $(\mathrm{E}$-value $=3.3 \mathrm{e}-331)$ motif of 15 nucleotides, which clearly contains a major 8 -mer core, essentially reflecting the previously described mammalian motif we found in the initial screening (Figure 1).

We next investigated the number of ribosomal genes containing single-nucleotide degenerate variants of the motif GUACAUUA and found that 224 (out of 472) of the 3' UTRs show a single mismatch to the motif. The average distance of the set of GUACAUUA singlenucleotide variants from the translation termination codon, as calculated for 194 unique hits, is $17.49 \mathrm{bp}$ (SD $=13.57)$ but decreases to $16.32 \mathrm{bp}(\mathrm{SD}=6.86)$ when two outliers (105 bp and 154 bp distant from the termination codon) are not accounted for in the analysis.

We noticed that only fifteen of the twenty-four possible single-nucleotide degenerate GUACAUUA variants were contained in the ribosomal 3' UTRs. In particular, we consistently detected 1) no motif variant with a base

Table 2 List of $\boldsymbol{P}$. tetraurelia non -ribosomal protein-coding genes that contain the 3' UTR motif GUACAUUA.

\begin{tabular}{cccc}
\hline GENE MODEL & MOLECULAR FUNCTION & BLAST score & BLAST E-value \\
\hline GSPATP00003019001 & Hypothetical protein & 60 & $7 \mathrm{e}-009$ \\
GSPATP00005027001 & Eukaryotic translation initiation factor & $7 \mathrm{e}-028$ \\
GSPATP00016207001 & Guanine nucleotide-binding protein & 229 & $8 \mathrm{e}-060$ \\
GSPATP00039830001 & Asparagine synthetase & 112 & $7 \mathrm{e}-025$ \\
GSPATP00000284001 & Asparagine synthetase & 263 & $1 \mathrm{e}-069$ \\
GSPATP00018093001 & AMP-binding enzyme & 337 & $8 \mathrm{e}-092$ \\
GSPATP00015762001 & Membrane transporter & 97 & $1 \mathrm{e}-019$ \\
GSPATP00033344001 & DNA-directed RNA polymerase I & 0.0 \\
GSPATP00026659001 & RNA-binding (PUF) protein & 980 & $1 \mathrm{e}-022$ \\
GSPATP00031576001 & Phosphatidylserine decarboxylase & 105 & $1 \mathrm{e}-039$ \\
GSPATP00005327001 & Nucleolar protein NOP58 & 162 & $4 \mathrm{e}-092$ \\
\hline
\end{tabular}




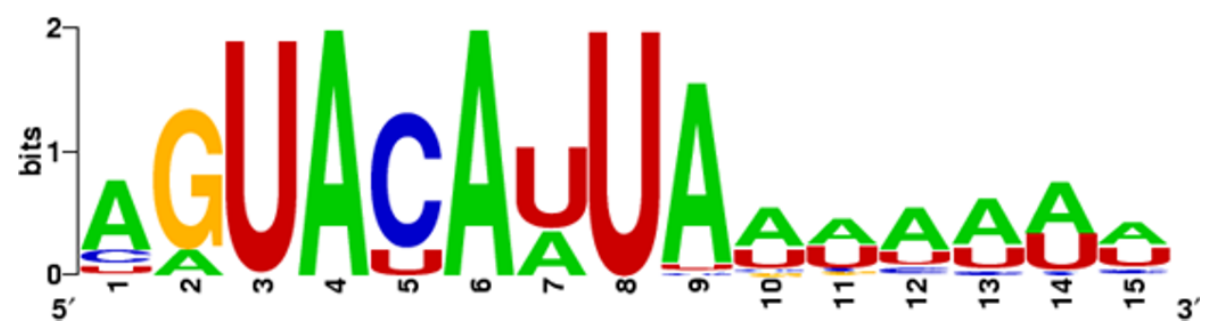

Figure 1 Nucleotide composition of the common 15-mer motif detected in the $3^{\prime}$ UTRs of ribosomal protein genes in P. tetraurelia. The core region (positions 2:9) reflects the highly preserved 3' UTR mammalian motif.

other than adenine at the third and fifth position, 2) no motif variant with either cytosine or guanine at the sixth position, and 3) a low frequency of adenine and cytosine as well as the absence of guanine at the seventh motif position (Figure 1).

We used the observations above, i.e., which nucleotides are either fixed or show highest frequency at each of the eight motif sites, to screen ribosomal 3' UTRs that contain neither GUACAUUA nor any of its singledegenerate variants, for a number of further degenerated, yet putatively functional, GUACAUUA variants. Specifically, we used the following degenerate motif (AGU)UA(CU)A(AU)U(AU), where the most degenerate nucleotides are presented within brackets, while the fixed nucleotides are in bold. Henceforth, we will refer to this set of motif variants using the consensus sequence DUAYAWUW, according to the appropriate IUPAC annotation. The consensus motif is positioned at a distance of $16.79 \mathrm{bp}(\mathrm{SD}=11.37$ ) from the translation termination codon. Sixty-two ribosomal genes (out of 472) did not contain the DUAYAWUW motif in their 3' UTR (Additional file 1).

\section{GUACAUUA and its variants appear conserved in Paramecium but not in other species}

We examined the degree of conservation of the detected ribosomal 3' UTR motif for four genes across multiple Paramecium species and verified that the ribosomal motif is conserved at this level-the inter-specific degree of sequence divergence was relatively low across the whole 3' UTRs however (data not shown). To further characterize the motif conservation across Paramecium species, we then BLASTed the motif-containing P. tetraurelia ribosomal 3' UTRs against the unassembled contigs of the newly sequenced (yet unreleased) Paramecium biaurelia macronuclear DNA. The net average pairwise divergence at nuclear silent sites between $P$. biaurelia and $P$. tetraurelia ranges between 0.30 and 0.45 (depending on the inclusion of outlier strains [14]). The analysis of the putative orthologous 3' UTRs in $P$. biaurelia suggests that the exact motif GUACAUUA is also conserved in this species.
Specifically, when we BLAST the 100 GUACAUUAcontaining ribosomal 3' UTRs against the $P$. biaurelia macronuclear DNA, we retrieve hits for 88 of them and in the 47 cases for which we could infer (putative) orthology, the motif was virtually always completely conserved (46 out of 47)(Additional file 1).

Finally, we screened the ribosomal 3' UTRs of four additional species, $T$. thermophila, $H$. sapiens, D. melanogaster and A. thaliana and found that none of these species contains an excess of the ribosomal motif conserved in P. tetraurelia (Table 3). For each of these four species, we further verified whether the motif GUACAUUA significantly hits 3' UTR sequences of gene classes that are not ribosomal. Gene Ontology annotation tools, g:Profiler [15] and GOEAST [16], and visual inspection hint at a slight enrichment for this motif in genes that code for proteins that are involved in: in $H$. sapiens, regulation of macromolecule biosynthesis and amino acid transport across the plasma membrane (respectively 67 and 5 proteins out of a total of 273 proteins); in D. melanogaster, cell projection organization (12 out of a total of 117 proteins); in A. thaliana, binding (32 out of a total of 92 proteins). No clear enrichment in a particular gene classis observed for T. thermophila.

Table 3 The number of occurrences of the GUACAUUA and its single nucleotide degenerate variants is examined in Paramecium and four additional species ( $T$. thermophila, D. melanogaster, A. thaliana and $H$. sapiens).

\begin{tabular}{cccc}
\hline Species & $\begin{array}{c}\text { Number } \\
\text { of } \\
\text { ribosomal } \\
\text { 3'UTRs }\end{array}$ & $\begin{array}{c}\text { GUACAUUA } \\
\text { (count) }\end{array}$ & $\begin{array}{c}\text { GUACAUUA single } \\
\text { nucleotide } \\
\text { degenerate } \\
\text { variants (count)† }\end{array}$ \\
\hline P. tetraurelia & 472 & $100(1)$ & $246(38)$ \\
T. thermophila & 81 & $1(1)$ & $16(35)$ \\
D. melanogaster & 186 & $1(2)$ & $19(37)$ \\
A. thaliana & 432 & $2(3)$ & $28(77)$ \\
H. sapiens & 283 & $1(3)$ & $43(83)$ \\
\hline
\end{tabular}

A motif is considered over- represented if its frequency in the set of ribosomal 3' UTRs is significantly higher $(P<0.01)$ than its frequency in the set of non-ribosomal $3^{\prime}$ UTRs. Average numbers of hits expected by chance arein parentheses. $\dagger=$ multiple hits are included 
Ribosomal genes that lack the motif or retain only uncommon 3' UTR motif variants are lost more rapidly The DUAYAWUW motif may serve a significant role for the biological activity of ribosomal genes in P. tetraurelia. Thus, we hypothesized that the protein-coding sequence of ribosomal genes that do not contain the 3' UTR DUAYAWUW motif may tend to show lower levels of evolutionary constraints compared to motifcontaining ribosomal genes.

We tested this hypothesis by taking advantage of the most recent whole genome duplications detected in $P$. tetraurelia. This duplication event is thought to have occurred in P. aurelia before the emergence of the 15 species-one of which is $P$. tetraurelia-that today belong to the $P$. aurelia complex $[17,18]$. We found that the $K_{\mathrm{a}} / \mathrm{K}_{\mathrm{s}}$ ratio is comparable between motif-free and motifcontaining genes. However, synonymous sites, but not non-synonymous sites, appear to evolve more rapidly between paralogous genes where at least one of the two genes lacks the 3' UTR DUAYAWUW motif $\left(t\right.$-test, $\mathrm{P}_{(1-}$ tail) $=0.0075)$ (Figure 2), and this faster sequence evolution extends to the 3' UTR sequences $\left(U\right.$-test, $\mathrm{P}_{(1 \text {-tail })}=$ $0.0365)$. We also observed that in paralogous genes containing DUAYAWUW, the motif sequence is highly conserved (i.e., the motif sequence is typically identical between paralogs) and that no correlation exists between motif diversity and $K_{s}$ (data not shown). Also, motif-containing ribosomal genes appear to retain a larger number of paralogs and to be more highly expressed compared to genes that only contain the rarest motif variants or no motif at all $(5.08 v s 4.00, \mathrm{M}-\mathrm{W}$ test, $P_{(1 \text {-tail })}=0.0014$; average ESTs number: 15.33 vs. 9.60 ,

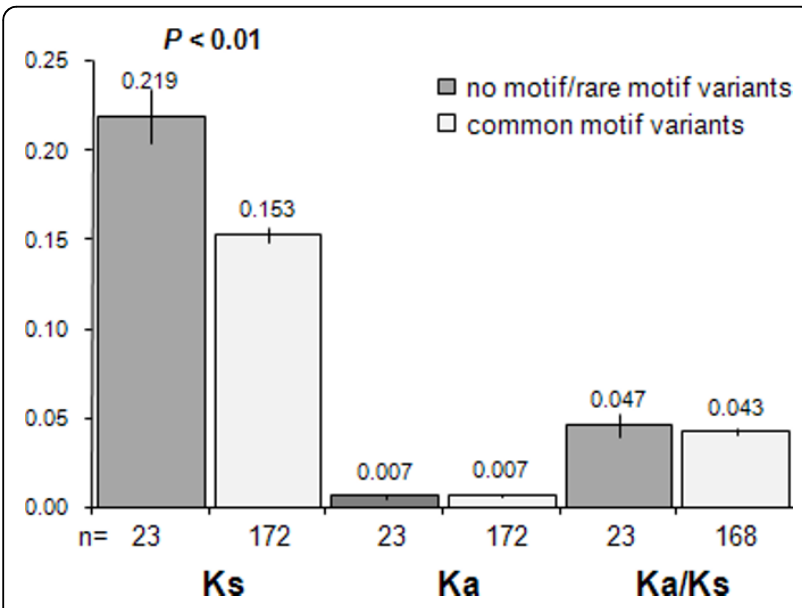

Figure 2 Average $K_{s}, K_{a}$ and $K_{a} / K_{s}$ values (and standard errors) estimated for pairs of duplicated ribosomal genes (as products of the most recent polyploidization event in $P$. tetraurelia). Average diversity values are examined between gene pairs where both copies contain the DUAYAWUW motif and gene pairs where at least one of the copies does not contain this motif.
$\mathrm{M}-\mathrm{W}$ test, $\left.P_{(1-\text { tail })}<0.001\right)$. Finally, the set of ribosomal genes that do not have a paralog that derives from the recent polyploidization event $(n=28)$ is enriched with motif-free genes. Specifically, the latter set contains 11 (of 47) motif-free genes and only 17 (of 373) motif-containing genes (Fisher's Exact Test, $\left.P_{(1-\text { tail })}=2.5 \times 10^{-4}\right)$.

\section{Can the detected conserved motif be a miRNA target?}

The conserved mammalian 3' UTRs motifs described by Xie et al. [2] show a distinct bias in DNA strand location, being preferentially conserved in the coding strand. This observation, jointly with both the 8-base motif length and the high frequency of an adenine as the ending nucleotide, led these authors to hypothesize a regulation activity associated with miRNAs, a hypothesis subsequently confirmed by experiments for a number of these motifs.

When we studied the strand specificity of the ribosomal motif (and motif variants), we found a pronounced abundance of the motif on the coding strand (it must be noted that a putative equally functional complementary motif is clearly just as abundant on the opposite strand). Indeed, the frequencies we reported above only refer to the presence of both GUACAUUA and its variants in the forward strand. When we searched for this motif on the complementary strand, we no longer observed an overrepresentation, and in fact we found no hits for the motif GUACAUUA on the complementary strand. The strand specificity we observe suggests that this 3' UTR motif acts at the RNA rather than at the DNA level and thus plays a role in post -transcriptional regulation.

How likely is it that the newly discovered motif is also a miRNA target in Paramecium? We addressed this question by taking advantage of some of the findings of a recent large-scale study of miRNAs in metazoans [19]: 1) uracil (U) is the most frequent nucleotide in mature miRNA sequences-being particularly enriched at the first and the ninth nucleotide positions, i.e., sites that immediately flank the miRNA "seed" region, which is believed to have a critical role in binding the target sequences; and 2) guanine $(G)$ is significantly depleted at position one.

Assuming that protozoans share the same or similar features with miRNAs in metazoans, an initial inspection of the sequence that is reverse complementary to the conserved ribosomal motif, i.e., UAAUGUAC (this sequence represents a portion of the putative miRNA sequence and includes, underlined, the seed region), is broadly consistent with features that are reminiscent of miRNA in metazoans (i.e., $U$ is enriched at the first position, where $\mathrm{G}$ is scarcely found). A closer analysis of the motif profile points to a higher level of resemblance. Specifically, the pictogram representation of the consensus motif presented in Figure 1 reveals the frequent 
occurrence of an A (or less frequently a $\mathrm{C}$ or a $\mathrm{U}$, but very rarely a $\mathrm{G}$ ) upstream of the consensus ribosomal motif, at position nine; such an enrichment in As is illustrated by the high frequency $(76 \%)$ with which an $\mathrm{A}$ immediately precedes the 100 GUACAUUA motifs that we found in the ribosomal 3' UTRs of P. tetraurelia (the frequency of adenines in the examined set of 3' UTRs is 44.1\%). This suggests that the putative complementary miRNA sequence is likely to contain a $U$ not only at position one but also at position nine, in agreement with what has been described for miRNA in metazoans.

If the complementary UAAUGUAC sequence is part of the miRNA sequence that targets the conserved ribosomal motif, then one could expect to find a region of the macronuclear DNA of $P$. tetraurelia containing this motif (along with its complementary version) and having a stable secondary structure that is typical of miRNA precursors. Alternatively the putative miRNA sequence could be located in the micronuclear DNA-in Paramecium there are two nuclei, the macronucleus (the somatic nucleus) and the micronucleus (the germline nucleus). In an attempt to identify a putative premiRNA, we screened the available $P$. tetraurelia macronuclear genome [20] for stem-loop structures containing the motif seed and its complementary version that are separated by up to 200 base pairs and flanked upstream and downstream by an arbitrary number of bases $(10$, 20 and $30 \mathrm{bps}$ ).

This screening yielded a total of 284 candidate premiRNAs. For each of these candidate elements we assessed the stability of the secondary structure by measuring: 1) the minimum free energies (MFE), as predicted by the RNAfold program [21]; the parameter AMFE ([MFE/length]"100)), which provides an estimate of stability that is not influenced by differences in RNA sequences length; and MFEI (AMFE/(G+C)\%), an index that appears to be valuable in distinguishing miRNAs from other coding and non-coding RNAs [22], and whose absolute values are consistently close or higher than 0.85 when experimentally confirmed miRNAs in metazoans are examined $[19,22]$. The study of these parameters across the candidate pre-miRNAs led to the identification of a putative pre-miRNA that, irrespective of the arbitrary number of flanking bases, showed consistently both low AMFE values $(-28.69 \pm 7.25)$, which are comparable to those detected for confirmed miRNAs in metazoans [19], and absolute values of MFEI close to or higher than $0.85(0.98 \pm 0.20)$. This candidate premiRNA, whose MFE structure is shown in Figure 3a, matches none of the currently available $P$. tetraurelia ESTs, has a single hit in the $P$. tetraurelia genome and is located in a region that is devoid of genes.

It is worth noting that a screening of the $P$. tetraurelia EST sequences led to the identification of an additional candidate pre-miRNA. This further putative pre-miRNA is detected in an EST that only partly matches the 3' end of a 605 ribosomal protein-coding gene. Specifically, while the 5' end sequence of this EST matches the ribosomal gene sequence as well as the homologous region of other ESTs that have been mapped to this gene, its 3' end sequence shows a unique profile that differs both from the sequence of the genomic DNA and the remaining ESTs that match this region. This EST's peculiar 3' end sequence is capable to form an extremely stable structure (AMFE average: $-43.61 \pm 9.26$; MFEI average: $-2.47 \pm 0.36$ ) (Figure $3 b$ ).

\section{Additional motifs in the $3^{\prime}$ UTRs of $P$. tetraurelia}

We finally screened the P. tetraurelia 3' UTRs for the presence of the hexanucleotide typically associated with polyadenylation in mammals (AAUAAA), and $k$-mer motifs that were previously found to be highly conserved in worms $(n=442)$ and flies $(n=497)$ [6]. The latter two sets of motifs include both verified and putative miRNA targets as well as GU and AU-rich elements and PUF-binding sites. We found that the AAUAAA motif hits the whole set of $P$. tetraurelia 3' UTRs 1780 times (the number expected by chance-obtained by screening 25 sets of randomly generated DNA sequences, whose length and nucleotide composition are identical to the 3' UTR sequences of the original dataset-is 1599 ( $\mathrm{SD}=27.81)$ ), and is located an average distance of 29.39 bp $(\mathrm{SD}=30.92)$ from the translation termination codon. We also found that several U-rich $k$ mers that are conserved in worm and/or flies, appear to be overrepresented in P. tetraurelia 3' UTRs. Aside from the GUACAAU and UGUACAUU motifs-which resemble the GUACAUUA sequence we describe above, and as such appear to be preferentially located in ribosomal 3' UTRs ( $64.5 \%$ and $43.3 \%$ respectively)-these overrepresented motifs are not clearly associated with a specific class of proteins, occur at various frequencies, and are located at different positions along the 3' UTRs (Table 4).

\section{Discussion and Conclusions}

A growing body of literature is providing critical information about innovative strategies for the identification of conserved cis-regulatory motifs [23-25] and about the regulatory interactions between conserved UTR sequences and miRNAs [2,6,12,13,26-30]. However, the evolutionary dynamics of conserved UTR motifs remain virtually unexplored.

In this article, we have reported the discovery and the characterization of a conserved 8-mer motif-GUACAUUA-as well as several of this motif's degenerated variants, in the 3' UTRs of $P$. tetraurelia ribosomal-protein genes. By studying the distribution frequency of 


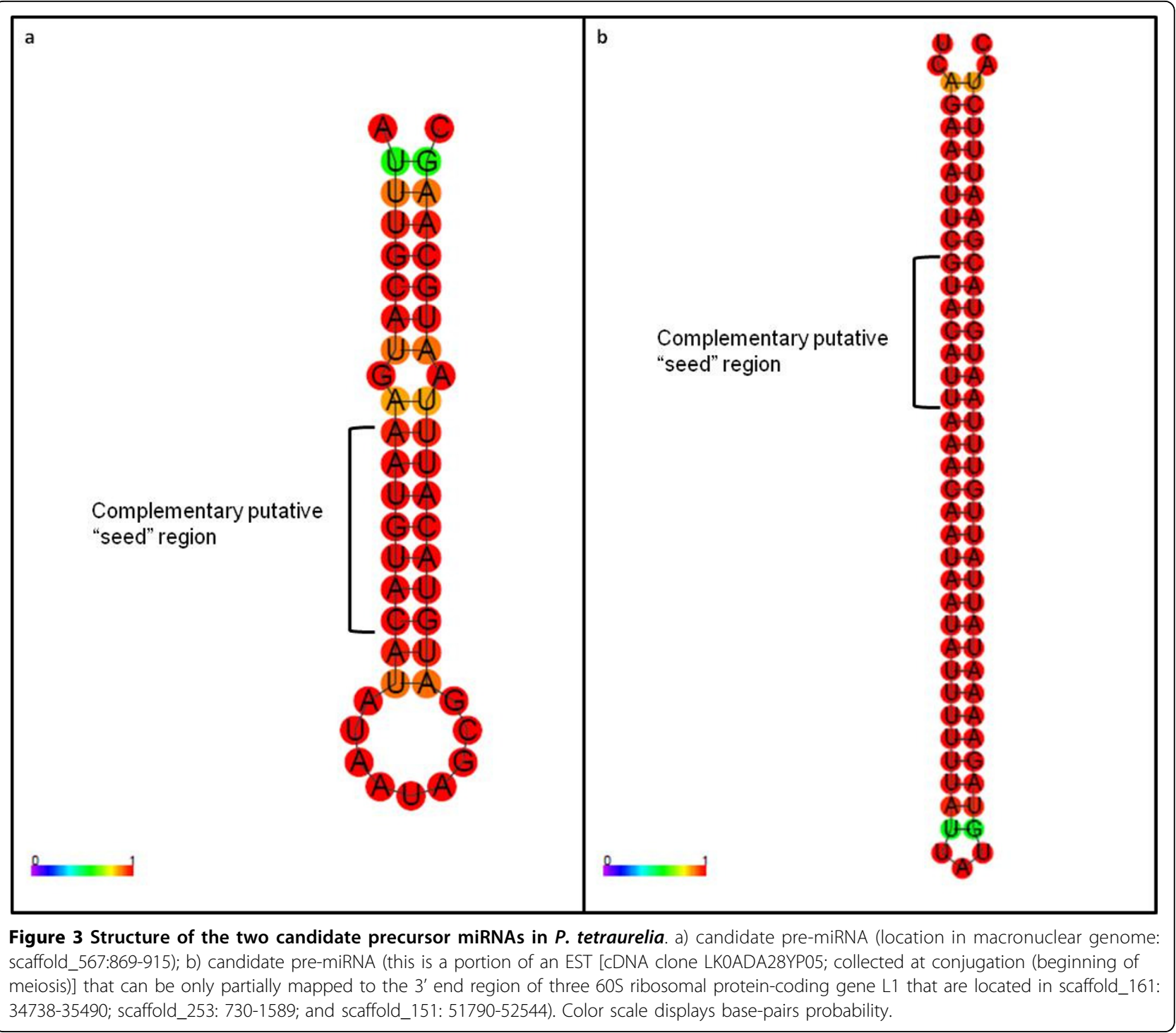

GUACAUUA single-nucleotide degenerated variants, we yielded the profile of the conserved consensus motif (DUAYAWUW), where four nucleotides are perfectly conserved and nucleotide frequencies at the remaining four positions tend to be skewed toward a single nucleotide (Figure 1). The region occupied by the preserved ribosomal sequence has overall a narrow size and the motif is relatively close to the translation termination codon (average distance of the start of the motif from termination codon is $17.9 \mathrm{bp}$ [SD = 7.30]). Both the positional range and the relative distance from the termination codon are smaller when compared to the corresponding values estimated for additional motifs we found to be overrepresented in P. tetraurelia3' UTRs (Table 4).

The study of the degree of conservation of the detected motif across Paramecium and non-Paramecium species shows that this motif is broadly conserved across multiple Paramecium species but is typically absent from the ribosomal genes of three multicellular organisms and, most notably, from another ciliate species (T. thermophila), which is distinct both morphologically and molecularly [31], yet an Oligohymenophoran like Paramecium. Unfortunately, the absence of genome sequence and EST information for species that are phylogenetically closer to Paramecium currently complicates the attempt to further trace back the evolutionary origin of the motif (but see below).

The hypothesis that the mere presence/lack of the motif can in some way influence (or be influenced by) the rate of protein-coding sequence evolution is not supported by the similar levels of constraints at non-synonymous sites observed for motif-free and motif-containing ribosomal genes (Figure 2). However, the study of $K_{\mathrm{s}}$ and 
Table 4 Highly conserved motifs in worms and/or flies [6] detected in P. tetraurelia $3^{\prime}$ UTRs.

\begin{tabular}{lccc}
\hline Motif & $\begin{array}{c}\text { Observed } \\
\text { hits }\end{array}$ & $\begin{array}{c}\text { Expected } \\
\text { hits }\end{array}$ & $\begin{array}{c}\text { Distance (bp) from } \\
\text { translation termination } \\
\text { codon }\end{array}$ \\
\hline UAAAUAAAU & 165 & $121(0.97)$ & $26.46(37.08)$ \\
UAUAUAUA & 689 & $246(3.56)$ & $23.52(25.23)$ \\
UGCAUUU & 146 & $64(1.76)$ & $35.46(44.36)$ \\
UGUGUAU & 106 & $53(0.99)$ & $26.98(32.07)$ \\
UUUUUAUA & 175 & $284(2.14)$ & $28.77(40.40)$ \\
UGUACAUU & 47 & $11(1)$ & $21.83(20.20)$ \\
GUACAAU & 109 & $33(2.46)$ & $21.55(20.10)$ \\
UCAAUAAA & 107 & $68(1.27)$ & $29.94(28.01)$ \\
UACUAAC & 12 & $35(0.81)$ & $32.33(19.81)$ \\
UUGCAUA & 130 & $61(2.59)$ & $28.75(41.93)$ \\
\hline
\end{tabular}

The list includes the top $10 \mathrm{k}$-mers for which the frequency of occurrence in $P$. tetraurelia 3' UTRs most deviate from the corresponding frequency expected by chance. Values in parentheses are standard deviations.

3' UTR sequence variation between duplicated ribosomal genes reveals higher rates of nucleotide substitutions at silent and 3' UTR sites in motif-free ribosomal genes compared with motif-containing genes. An explanation for the association between the lack of motif and the faster evolution at silent and 3' UTR sites may be non-biological. Specifically, the motif-free ribosomal genes we used to estimate nucleotide variation may not all be the most recent paralogs, as initially assumed. A number of gene duplicates in the set of motif-free genes could result from independent (and temporally distant) events of WGD, but be considered as most recent copies if their true closely related copies have gone lost. Consistent with this hypothesis, we find that motif-free ribosomal genes retain significantly fewer paralogs and are lost more often relative to motif-containing genes. Also, $K_{\mathrm{s}}$ estimates between motif-free gene copies tend to be high $(>0.25)$ when only two duplicates are detected in the genome, a condition that may more easily lead to classify incorrectly the gene copies as recent duplicates. Finally, as the identification of the WGD paralogs involved also the study of the synteny between blocks of duplicated genes [20], by visual inspection we find that similar features can be shared between motif-free ribosomal duplicates and their flanking genes, i.e., flanking genes retain also only one paralog and/or show comparable high levels of divergence at silent sites (data not shown). The latter observation implies that the hypothetical incorrect assignment of recent paralogy could not only involve the motif-free ribosomal genes but extend also to their flanking genes.

Finally, the observation that the GUACAUUA sequence is typically located on the forward DNA strand is consistent with the idea that this motif is involved in post-transcriptional regulation-although the alternative explanation that the motif is a DNA binding protein motif cannot be ruled out for now. Three observations hint at the possibility that the newly described motif plays a role in modulating expression of the host gene. First, motif-containing and motif-free genes are differentially expressed, with motif-containing genes being more highly expressed compared to motif-free genes, a finding that along with the higher levels of retention described for motif-containing genes is reminiscent of the reported positive correlation between gene retention and gene expression in Paramecium [32]. Second, the GUACAUUA sequence is reminiscent of the PUF-binding site (UGUAnAUA), and PUF proteins-a family of mRNA-binding proteins-are known to repress gene expression, either by inhibiting the translation or by enhancing the decay of target mRNAs $[33,34]$. Third, the GUACAUUA sequence closely resembles a conserved 3' UTR motif in yeast, UGUAUAUUA, that mediates the destabilization of the host mRNA [8]. Intriguingly, this yeast motif is also enriched in ribosomal genes, and has a mammalian counterpart that is the target of a miRNA, miR-381 [8]. The implications of the latter observations are twofold: 1) the GUACAUUA motif was probably not gained independently in mammals and Paramecium but emerged in the common ancestor of the surveyed species and underwent either secondary loss or switches in expressed genes; the GUACAUUA motif may too be a binding site for a miRNA, which would be expected to have co-evolved with the core motif. While an experimental validation is clearly needed to provide any solid support for the latter hypothesis, the possibility of a connection between the motif and miRNAs is twofold intriguing: 1) the putatively regulated genes (i.e., ribosomal protein-coding genes) would not be lowly or only moderately expressed genes, as genes that are commonly thought to be typical targets of miRNAs, and 2) aside from small RNAs that are involved in the definition of the new macronucleus [35], and a class of short RNAs that are involved in post-transcriptional genesilencing [36], miRNAs have never been described in ciliates.

\section{Methods}

\section{Motif characterization}

We investigated the presence of conserved signal sequences in the 3' untranslated regions of Paramecium, by extracting 7647 annotated 3' UTR sequences from the $P$. tetraurelia genome database [37], and screening these sequences for motifs that have been already catalogued for other species (a flow chart is provided in Additional file 2). In particular, we used 540 8-mers that were previously identified in mammalian 3' UTRs [2], as well as 442 and $497 k$-mers that are highly conserved in worms and flies respectively [6]. The procedure of gene, 
and thus of UTR, annotation in $P$. tetraurelia is described in Aury et al. [20] and takes advantage of cDNA libraries that include gene transcripts detected at six different physiological conditions/developmental stages. The average length calculated for the studied 3' UTRs is $52.1 \mathrm{bp}(\mathrm{CV}=0.68)$.

As conserved signals may be more likely to be shared among genes coding for proteins that have similar functions, we characterized the molecular role of $P$. tetraurelia genes having an annotated 3' UTR, by BLASTing the corresponding protein sequences against the UNIPROT database [38]. We used an E-value cut-off of $10^{-7}$ and gap opening and gap extension penalties equal to 10 and 1 respectively. We assigned a given molecular function to each of the examined $P$. tetraurelia proteins, according to the information drawn from the BLAST first best hit.

We used the motif discovery MEME software [39] to verify the exclusive presence and the most likely width of the conserved motif we discovered in genes coding for ribosomal proteins (see below) and Weblogo [40] to produce a graphical view of the degree of conservation at every site.

\section{Interspecific conservation of the motif}

We performed PCR and DNA sequencing to assess the level of conservation of the detected $P$. tetraurelia ribosomal motif across multiple species of the $P$. aurelia complex and a species closely related to the $P$. aurelia species complex, P. caudatum. We designed PCR primers using the coding regions of two adjacent genes (the upstream of which being a ribosomal protein gene) and obtained PCR products spanning the intervening ribosomal 3' UTR. Due to difficulties in DNA amplification across the whole set of (or most) species surveyed, the analysis across these relatively diverged species [14] produced successful results only for a limited number of 3' UTRs $(\mathrm{n}=4)$ (data not shown). We calculated the level of sequence divergence $(d)$ along the entire 3' UTR using the Maximum Composite Likelihood method implemented in the software MEGA 4.0 [41].

To further verify the degree of evolutionary conservation of the motif detected in $P$. tetraurelia, we next surveyed the 3' UTRs annotated for ribosomal protein genes of four non-Paramecium species (Tetrahymena thermophila, Homo sapiens, Drosophila melanogaster and Arabidopsis thaliana). It is worth stressing that the 8-mer we discovered in the P. tetraurelia 3' UTRs, as well as the remainder of conserved mammalian motifs we used for our initial screening, had not been explicitly associated with specific gene functions in mammals. Using the same procedure described above to infer the molecular function of $P$. tetraurelia genes, we collected the following number of unique ribosomal genes (i.e. isoforms are not counted) for each of the additional species surveyed: 142 (T. thermophila), 282 (H. sapiens), 176 (D. melanogaster), 521 (A. thaliana). For each of the latter three sets we directly extracted the annotated 3' UTRs. In T. thermophila, where 3' UTRs are not annotated, putative 3 ' UTR sequences were retrieved by cropping $500 \mathrm{bp}$ of genomic DNA, downstream of each ribosomal gene, and blasting the cropped region against the whole set of $T$. thermophila ESTs. The EST hit that extends most downstream of each of the translation termination codons was used as 3' UTR for this study.

\section{Levels of sequence divergence between recently duplicated ribosomal genes}

To verify whether a correlation exists between the evolution of the motif sequence and that of the coding sequence of the gene that contains it, we estimated the level of sequence divergence between recently duplicated $P$. tetraurelia macronuclear ribosomal protein-coding genes (as derived from the most recent event of whole genome duplication (WGD) in P. tetraurelia). Specifically, we grouped separately pairs where both genes contain the discovered conserved motif and pairs where at least one of the two genes was motif-free. We verified the absence of the conserved motif in all the motif-free genes that only contained a short (i.e. presumably incomplete) annotated 3' UTR. In one case we found that the $9 \mathrm{bp}$ annotated 3' UTR of the gene model GSPATP00010990001 contained the motif within the following $10 \mathrm{bp}$ and we included this gene in the set of motif-containing genes.

For each of the ribosomal genes in P. tetraurelia, we retrieved the most recent paralog according to the 'ALL-against-ALL' Blast analysis and the analysis of the synteny between blocks of duplicated genes performed by Aury et al. [20]. We aligned the duplicate sequences with ClustalW [42], verified the existence of indels, suspicious introns, correct UTRs and gene predictions (see below), and performed manual editing if needed. We used the Kumar method implemented in the program MEGA 4.0 to estimate the average $K_{a}, K_{s}$ and $K_{a} / K_{s}$. We used both the $t$-test and the Mann-Whitney $U$-test to assess the significance of the observed differences. We performed the $t$-test after square root transforming the raw data and testing both for normality ( $K-S$ test) and for equality of variances (Levene's test). After data transformation, a normal distribution could be obtained consistently only for the $K_{s}$ estimates.

We also estimated nucleotide diversities (corrected for multiple substitutions [43]) for all pairs of paralogous ribosomal 3' UTRs, after the removal of the 8-mer motif. When 3' UTRs contained more than one motif copy, we randomly removed one of the copies. 


\section{Inspection of the ribosomal genes' coding sequences}

To calculate estimates of levels of sequence divergence we used alignments of putatively functional genes, i.e., we discarded pseudogenes, a condition that is not uncommon for genes that code for ribosomal proteins. The vast majority of alignments produced by ClustalW were highly reliable-no manual editing was needed, and the sequences always had virtually identical length. In nine cases (one in the motif-free set and eight in the motif-containing set), the presence of indels not verified by ESTs or a high variability, both in the very 5 ' end of the gene sequences, complicated the local alignment. In these cases, we only aligned the corresponding sequences starting from the first ESTs verified site when ESTs were available, or from the site where the alignment started to be unambiguous, when ESTs were not available. We include these gene pairs in our analysis and verified that their removal did not affect our conclusions.

Further, we detected a limited number of non-framepreserving indels. Such mutations typically lead to incorrect introns, UTR or gene predictions and might reflect a pseudogenization event. We verified and rejected the occurrence of every indel (presumably deriving by sequencing errors), by examining the corresponding ESTs and re-estimated diversity after reintroducing the erroneously eliminated coding regions. When ESTs were not available, we excluded the gene pairs containing the non-frame-preserving indel(s) from the analysis, as these genes may represent pseudogenes.

Finally, in an additional attempt to detect pseudogenes in our study, we examined intronless genes. The reason for the latter analysis is that pseudogenes could arise after an event of reverse transcription followed by reinsertion into the genomic DNA (processed pseudogenes). We found only one gene with no introns. This gene, for which no ESTs are available, is a motif-containing ribosomal gene and shows a relatively high $K_{\mathrm{a}}$ value $\left(K_{\mathrm{a}}=0.10\right)$ and a predicted premature translation termination codon. We removed the corresponding gene pair from our analysis.

Additional file 1: Lists of motif-containing and motif-free ribosomal genes. The lists include the name of the ribosomal genes and their corresponding 3'UTR sequences with/without the GUACAUUA motif, with the single-nucleotide degenerate GUACAUUA variants and the consensus DUAYAWUW motif. The list includes also the (putative) orthologous 3'UTR sequences from $P$. biaurelia.

Additional file 2: Flow-chart. Flow-chart indicating the bioinformatic procedures used in the study.

\section{Acknowledgements}

We thank Eric Meyer, Jean-François Gout and two anonymous reviewers for their comments and suggestions on how to improve our study. This work was supported by MetaCyte funding from the Lilly Foundation to Indiana University and the National Science Foundation grantsEF -0827411 to M.L.

\section{Authors' contributions}

FC conceived and conducted the study. FC and ML wrote the manuscript.

Received: 5 December 2009 Accepted: 4 May 2010

Published: 4 May 2010

\section{References}

1. Deshler JO, Highett MI, Abramson T, Schnapp BJ: A highly conserved RNAbinding protein for cytoplasmic mRNA localization in vertebrates. Curr Biol 1998, 8(9):489-496.

2. Xie X, Lu J, Kulbokas EJ, Golub TR, Mootha V, Lindblad-Toh K, Lander ES, Kellis M: Systematic discovery of regulatory motifs in human promoters and 3' UTRs by comparison of several mammals. Nature 2005, 434(7031):338-345.

3. Gerber AP, Luschnig S, Krasnow MA, Brown PO, Herschlag D: Genome-wide identification of mRNAs associated with the translational regulator PUMILIO in Drosophila melanogaster. Proc Natl Acad Sci USA 2006, 103(12):4487-4492

4. Lai EC, Burks C, Posakony JW: The K box, a conserved 3' UTR sequence motif, negatively regulates accumulation of enhancer of split complex transcripts. Development 1998, 125(20):4077-4088.

5. Parsch J, Stephan W, Tanda S: A highly conserved sequence in the $\mathbf{3}^{\prime}$ untranslated region of the Drosophila Adh gene plays a functional role in Adh expression. Genetics 1999, 151(2):667-674.

6. Chan CS, Elemento O, Tavazoie S: Revealing posttranscriptional regulatory elements through network-level conservation. PLoS Comput Biol 2005, 1(7):e69.

7. Hajarnavis A, Durbin R: A conserved sequence motif in $3^{\prime}$ untranslated regions of ribosomal protein mRNAs in nematodes. RNA 2006, 12(10):1786-1789.

8. Shalgi R, Lapidot M, Shamir R, Pilpel Y: A catalog of stability-associated sequence elements in 3' UTRs of yeast mRNAs. Genome Biol 2005, 6(10):R86.

9. Chen Q, Adams CC, Usack L, Yang J, Monde RA, Stern DB: An AU-rich element in the $3^{\prime}$ untranslated region of the spinach chloroplast petD gene participates in sequence-specific RNA-protein complex formation. Mol Cell Biol 1995, 15(4):2010-2018.

10. Murray A, Fu C, Habibi G, McMaster WR: Regions in the 3' untranslated region confer stage-specific expression to the Leishmania mexicana a600 -4 gene. Mol Biochem Parasitol 2007, 153(2):125-132.

11. Wu J, Sieglaff DH, Gervin J, Xie XS: Discovering regulatory motifs in the Plasmodium genome using comparative genomics. Bioinformatics 2008, 24(17):1843-1849.

12. Lim LP, Lau NC, Garrett-Engele P, Grimson A, Schelter JM, Castle J, Bartel DP, Linsley PS, Johnson JM: Microarray analysis shows that some microRNAs downregulate large numbers of target mRNAs. Nature 2005, 433(7027):769-773.

13. Chang YM, Juan HF, Lee TY, Chang YY, Yeh YM, Li WH, Shih AC: Prediction of human miRNAs using tissue-selective motifs in 3' UTRs. Proc Natl Acad Sci USA 2008, 105(44):17061-17066.

14. Catania F, Wurmser F, Potekhin AA, Przybos E, Lynch M: Genetic diversity in the Paramecium aurelia species complex. Mol Biol Evol 2009, 26(2):421-431

15. Reimand J, Kull M, Peterson H, Hansen J, Vilo J: g:Profiler-a web-based toolset for functional profiling of gene lists from large-scale experiments. Nucleic Acids Res 2007, 35 Web Server: W193-200.

16. Zheng Q, Wang XJ: GOEAST: a web-based software toolkit for Gene Ontology enrichment analysis. Nucleic Acids Res 2008, , 36 Web Server: W358-363.

17. Aufderheide KJ, Daggett PM, Nerad TA: Paramecium-Sonneborni N -Sp, a New Member of the Paramecium-Aurelia Species -Complex. Journal of Protozoology 1983, 30(1):128-131.

18. Sonneborn TM: The Paramecium-Aurelia Complex of 14 Sibling Species. Transactions of the American Microscopical Society 1975, 94(2):155-178.

19. Zhang B, Stellwag EJ, Pan X: Large-scale genome analysis reveals unique features of microRNAs. Gene 2009, 443(1-2):100-109.

20. Aury JM, Jaillon O, Duret L, Noel B, Jubin C, Porcel BM, Segurens B, Daubin V, Anthouard V, Aiach N, et al: Global trends of whole-genome duplications revealed by the ciliate Paramecium tetraurelia. Nature 2006 444(7116):171-178.

21. Gruber AR, Lorenz R, Bernhart SH, Neubock R, Hofacker IL: The Vienna RNA websuite. Nucleic Acids Res 2008, 36 Web Server: W70-74 
22. Zhang BH, Pan XP, Cox SB, Cobb GP, Anderson TA: Evidence that miRNAs are different from other RNAs. Cell Mol Life Sci 2006, 63(2):246-254.

23. Cora D, Di Cunto F, Caselle M, Provero P: Identification of candidate regulatory sequences in mammalian $3^{\prime}$ UTRs by statistical analysis of oligonucleotide distributions. BMC Bioinformatics 2007, 8:174.

24. Andken BB, Lim I, Benson G, Vincent JJ, Ferenc MT, Heinrich B, Jarzylo LA, Man HY, Deshler JO: $3^{\prime}$-UTR SIRF: a database for identifying clusters of whort interspersed repeats in $3^{\prime}$ untranslated regions. BMC Bioinformatics 2007, 8:274.

25. Ivan $\mathrm{A}$, Halfon MS, Sinha S: Computational discovery of cis-regulatory modules in Drosophila without prior knowledge of motifs. Genome Biol 2008, 9(1):R22

26. Gu J, Fu H, Zhang X, Li Y: Identifications of conserved 7-mers in 3'-UTRs and microRNAs in Drosophila. BMC Bioinformatics 2007, 8:432

27. Lee RC, Feinbaum RL, Ambros V: The C. elegans heterochronic gene lin -4 encodes small RNAs with antisense complementarity to lin-14. Cell 1993, 75(5):843-854

28. Wightman B, Ha I, Ruvkun G: Posttranscriptional regulation of the heterochronic gene lin-14 by lin-4 mediates temporal pattern formation in C. elegans. Cell 1993, 75(5):855-862.

29. Rhoades MW, Reinhart BJ, Lim LP, Burge CB, Bartel B, Bartel DP: Prediction of plant microRNA targets. Cell 2002, 110(4):513-520.

30. Reinhart BJ, Slack FJ, Basson M, Pasquinelli AE, Bettinger JC, Rougvie AE, Horvitz HR, Ruvkun G: The 21-nucleotide let-7 RNA regulates developmental timing in Caenorhabditis elegans. Nature 2000 403(6772):901-906

31. Tourancheau AB, Villalobo E, Tsao N, Torres A, Pearlman RE: Protein coding gene trees in ciliates: comparison with rRNA-based phylogenies. Mol Phylogenet Evol 1998, 10(3):299-309.

32. Gout JF, Duret L, Kahn D: Differential retention of metabolic genes following whole-genome duplication. Mol Biol Evol 2009, 26(5):1067-1072.

33. Gerber AP, Herschlag D, Brown PO: Extensive association of functionally and cytotopically related mRNAs with Puf family RNA-binding proteins in yeast. PLOS Biol 2004, 2(3):E79.

34. Graber JH: Variations in yeast 3'-processing cis-elements correlate with transcript stability. Trends Genet 2003, 19(9):473-476.

35. Lepere G, Betermier M, Meyer E, Duharcourt S: Maternal noncoding transcripts antagonize the targeting of DNA elimination by scanRNAs in Paramecium tetraurelia. Genes Dev 2008, 22(11):1501-1512.

36. Lepere G, Nowacki M, Serrano V, Gout JF, Guglielmi G, Duharcourt S, Meyer E: Silencing-associated and meiosis-specific small RNA pathways in Paramecium tetraurelia. Nucleic Acids Res 2009, 37(3):903-915.

37. Arnaiz O, Cain S, Cohen J, Sperling L: ParameciumDB: a community resource that integrates the Paramecium tetraurelia genome sequence with genetic data. Nucleic Acids Res 2007, , 35 Database: D439-444.

38. Apweiler R, Bairoch A, Wu CH, Barker WC, Boeckmann B, Ferro S, Gasteiger E, Huang H, Lopez R, Magrane M, et al: UniProt: the Universal Protein knowledgebase. Nucleic Acids Res 2004, 32 Database: D115-119.

39. Bailey $T L$, Elkan C: Unsupervised Learning of Multiple Motifs in Biopolymers Using Expectation Maximization. Machine Learning 1995, 21(1-2):51-80.

40. Crooks GE, Hon G, Chandonia JM, Brenner SE: WebLogo: a sequence logo generator. Genome Res 2004, 14(6):1188-1190.

41. Tamura K, Dudley J, Nei M, Kumar S: MEGA4: Molecular Evolutionary Genetics Analysis (MEGA) software version 4.0. Mol Biol Evol 2007, 24(8):1596-1599.

42. Thompson JD, Higgins DG, Gibson TJ: CLUSTAL W: improving the sensitivity of progressive multiple sequence alignment through sequence weighting, position-specific gap penalties and weight matrix choice. Nucleic Acids Res 1994, 22(22):4673-4680.

43. Jukes TH, Cantor CR: Mammalian Protein Metabolism. Academic Press, New York 1969.

doi:10.1186/1471-2148-10-129

Cite this article as: Catania and Lynch: Evolutionary dynamics of a conserved sequence motif in the ribosomal genes of the ciliate Paramecium. BMC Evolutionary Biology 2010 10:129.

\section{Submit your next manuscript to BioMed Central and take full advantage of:}

- Convenient online submission

- Thorough peer review

- No space constraints or color figure charges

- Immediate publication on acceptance

- Inclusion in PubMed, CAS, Scopus and Google Scholar

- Research which is freely available for redistribution

Submit your manuscript at www.biomedcentral.com/submit
Ciomed Central 